\title{
Pleiotropy of tissue-specific growth factors: from neurons to vessels via the bone marrow
}

\author{
Dan G. Duda and Rakesh K. Jain
}

Edwin L. Steele Laboratory, Department of Radiation Oncology, Massachusetts General Hospital and Harvard Medical School, Boston, Massachusetts, USA.

\begin{abstract}
Recent evidence has demonstrated that endothelial-specific growth factors affect the development of apparently unrelated organs and cells. Expanding this evidence further, new findings in this issue of the JCI show that neurotrophic factors can affect neovascularization (see the related article beginning on page 653). Neurotrophic factors achieve proangiogenic effects not only by directly affecting endothelial cells, but also by recruiting hematopoietic precursors. Further understanding of the biology of angiogenic factors, as well as of the function of hematopoietic cells in tissue neovascularization, will lead to improved therapeutic strategies for the treatment of diseases ranging from ischemia to cancer.
\end{abstract}

Growth factors that were once considered tissue specific can have unexpectedly broad effects. For example, signals from endothelial growth factors govern not only tissue neovascularization, but also the development and function of different organs such as the pancreas (1), liver (2), fat (3), and hematopoietic tissues (4). Similarly complex relationships have been found in the nervous system (5). VEGF (also known as VEGF-A) is a critical factor in angiogenesis during development and disease (6); nonetheless, it has recently been shown to be neuroprotective in a rat model of amyotrophic lateral sclerosis (a neurodegenerative disease causing a progressive loss of motor neurons). This finding provides an unexpected new therapeutic platform for the treatment of this debilitating disease (7). Some elegant work in mutant mouse models has also demonstrated that factors such as brain-derived neurotrophic factor (BDNF; ref. 8) and receptors involved in axon guidance (9) play a crucial role in neovascularization during embryonic development.

\section{Neurotrophic factors promote adult neovascularization}

In this issue of the JCI, Kermani et al. (10) describe, we believe for the first time, the

Nonstandard abbreviations used: Ang1, angiopoietin 1 ; BDNF, brain-derived neurotrophic factor; BMDC, bone marrow-derived cell; NT-4, neurotrophin-4; PlGF, placenta-derived growth factor; TrkB, BDNF receptor tyrosine kinase.

Conflict of interest: Rakesh K. Jain is a consultant to AstraZeneca Pharmaceuticals LP.

Citation for this article: J. Clin. Invest. 115:596-598 (2005). doi:10.1172/JCI200524511. effects of BDNF and neurotrophin-4 (NT-4) on neovascularization in adult mice. The proangiogenic effects of these hypoxia-responsive neurotrophic factors are comparable to those of VEGF but are independent of the VEGF receptor 2 pathway. In part, these effects are exerted directly through the cognate receptor for the neurotrophic factors, BDNF receptor tyrosine kinase (TrkB) that is expressed on subsets of endothelial cells. More strikingly, the authors show that BDNF is similar to other angiogenic factors - such as VEGF, angiopoietin 1 (Ang1), and placenta-derived growth factor (PIGF) $(4,11)$ - in its ability to affect the hematopoietic system by mobilizing precursor cells (Figure 1). Recruitment of these precursor cells promoted neovascularization (Figure 2) and alleviated limb ischemia.

This work establishes that neurotrophins are novel proangiogenic factors. The authors report (10) that BDNF does not induce the recruitment of pericytes to blood vessels (an important step in the stabilization and maturation of new vasculature), but rather promotes the recruitment of myeloid progenitor cells of unknown function. These findings warrant further investigation of the role and therapeutic potential of these factors in the treatment of diseases ranging from cancer to cardiac ischemia. Moreover, they describe yet another facet of the intricate relationship between hematopoietic cells and tissue neovascularization. Understanding the molecular and cellular underpinnings of this interaction may prove to be crucial for both regenerative medicine and cancer therapy.

\section{Bone marrow-derived cell-mediated neovascularization}

The recruitment of hematopoietic bone marrow-derived cells (BMDCs) and the infiltration of these cells into diseased tissue is often massive in scale, particularly in tumors. But whether the presence of these cells is indicative of a response of the immune system directed against the cancer cells or of a nonspecific inflammatory response that favors tumor growth remains to be determined (12). Alternatively, if the immune cell infiltrate in tumors is the result of the exponential expansion of precursors (i.e., stem and/or progenitor cells), the function of BMDCs in the tumor might be significantly different. Previous reports have demonstrated the ability of hematopoietic cell precursors to secrete factors that modulate tissue vascularization, including factors such as Ang2 (13) and MMPs (14), which affect vessel wall stability. Others have shown that myeloid cells incorporate directly into the vasculature and acquire an endothelial or mural cell phenotype (15-17) (Figure 2). Some of these findings have led to clinical trials for bone marrow-mononuclear cell infusion to induce therapeutic angiogenesis, with encouraging results (18). Increasing evidence suggests that the contribution of hematopoietic stem cells and myeloid progenitor cells is, in fact, critical for new vessel formation in tumors (19). However, the mechanism of action and the degree of plasticity of these cells are not sufficiently understood. Once they have homed to the tumor, multipotent BMDCs have the potential to modulate neovascularization and to become part of all 3 nonmalignant tumor compartments: hematopoietic, endothelial, and mesenchymal (20). Both the relative contribution of different BMDCs to solid tumors and the timing of their incorporation remain unclear. The difficulty of assessing the relative contribution of each lineage of BMDCs to tumor neovascularization has led to debate and hampered the identification of new clinical targets, inhibiting clinical translation of the progress made in preclinical models. 


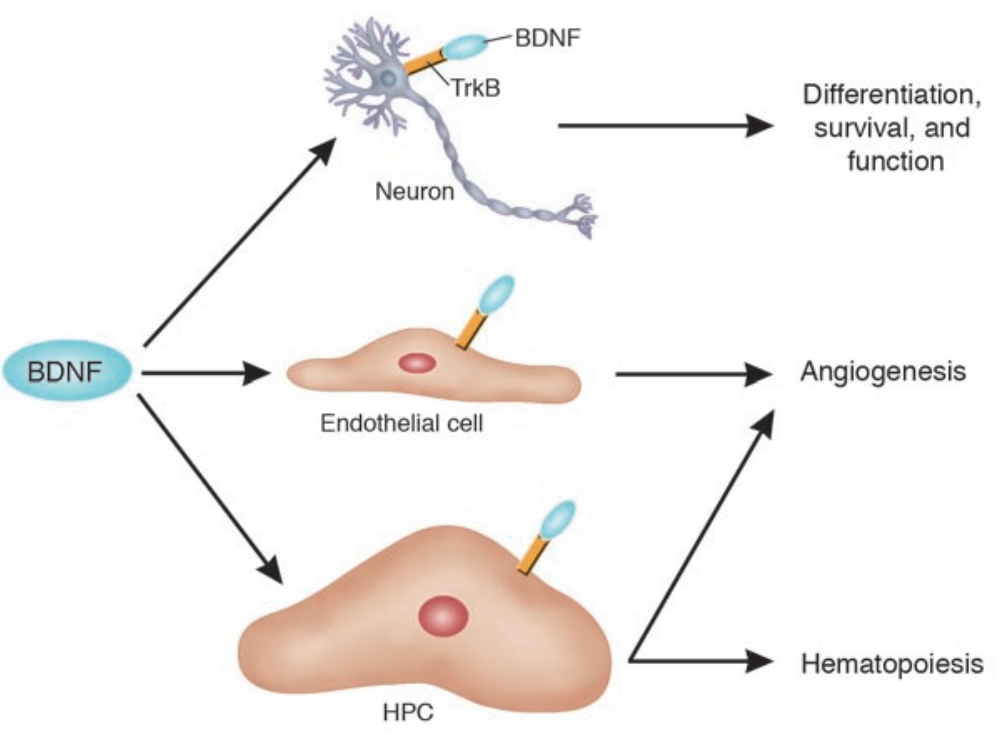

\section{Future directions}

These exciting findings have broad implications and raise a number of critical questions. BDNF may affect multiple cell types, similar to VEGF, PlGF, and Ang1 (Figure 2). It may therefore be possible to take advantage of the pleiotropy of all of these factors in order to establish surrogate markers for the determination of the biological effects of their blockade or overexpression both in preclinical models and in patients. Whether the effects of BDNF on adult vasculature are organ specific, and what effect BDNF has on myocardial revascularization or tumor growth, also needs to be investigated. Furthermore, what is the safest and most efficient combination of growth factors that generates collateral vessels and mature, long-lasting new vessels? In general, the mobilization of cells from the bone marrow requires a transient overexpression of factors such as BDNF. How well does this temporal and spatial overexpression of growth factors mimic tissue ischemia and/or tumor growth? Are myeloid precursors recruited to the wrong site by tumor-expressed chemoattractants and

\section{Figure 1}

The functional pleiotropy of BDNF extends beyond the nervous system. BDNF, similar to NT-4, exerts its complex signaling effects via TrkB that is present on neurons. These effects include the modulation of neuronal differentiation, survival, and function. In this issue of the $\mathrm{JCl}$, Kermani et al. (10) report a more complex role for this neurotrophic factor: BDNF can mobilize $\mathrm{TrkB}^{+}$hematopoietic precursor cells (HPCs) for both hematopoiesis and tissue neovascularization. In addition, BDNF can promote angiogenesis by directly interacting with TrkB expressed on ECs. local activation of the endothelium? What are the functions of VEGF and angiopoietin receptors in myeloid precursor cells? It has recently been suggested that bone marrow precursors for nonhematopoietic cells, such as endothelial and mesenchymal cells, participate in or even initiate tumor growth (21-23). It will be interesting to investigate whether their contribution is the result of a high degree of plasticity that arises in the context of sustained injury, or if the bone marrow contains multiple, distinct precursors. In addition, determining the relative contri-

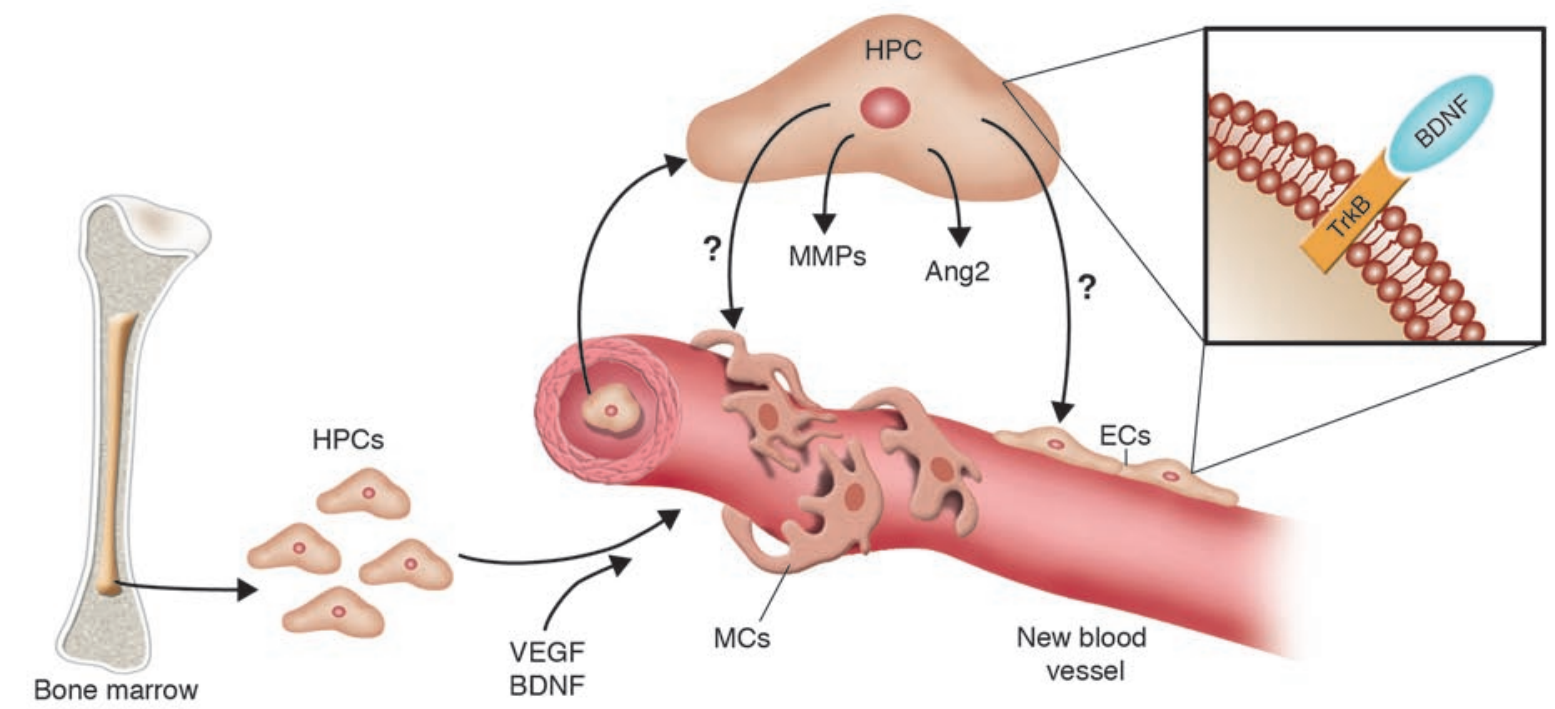

Figure 2

Cellular and molecular mechanisms of BDNF-induced neovascularization. BDNF was recently implicated in new vessel formation, in both mouse embryos (8) and adult mice (10). In adults, the formation of new vessels in response to BDNF overexpression is the result of both direct effects on TrkB expressed by tissue-resident ECs and the recruitment of TrkB+VEGFR2 ${ }^{+} \mathrm{CD} 11 \mathrm{~b}^{+} \mathrm{Sca} 1^{+}$myeloid HPCs. The latter cells may indirectly promote neovascularization by releasing various factors, including Ang2 and MMPs. Nevertheless, a direct involvement of myeloid HPCs in vessel formation cannot be excluded, as they also have the potential to acquire an EC or mural cell (MC) phenotype. 
bution of these cell populations in cancer and whether these cells use similar mobilization and homing mechanisms will be of great value. Also, how can we target BMDC-dependent angiogenesis while maintaining the defense mechanisms provided by hematopoietic cells? This question is of critical importance since many cancer patients receive treatments that promote recovery of hematopoiesis (such as infusion of GM-CSF or, more recently, mesenchymal stem cells) during cytotoxic treatments. Finally, while it is important to understand the roles played by BMDCs in tumor progression, a major challenge in the clinic is to abrogate the relapse of tumors that have previously been treated. If BMDCs play a significant role in the progression of cancer (24), then it is crucial to explore their role in tumor relapse.

The last decade has seen unprecedented breakthroughs in our understanding of the biology of angiogenic growth factors, culminating recently in the first successful clinical trial for an agent that targets one such growth factor in cancer patients (25). These advances have coincided with these new discoveries about the biology of BMDCs and their role in cancer, raising the hope that new antiangiogenic and proangiogenic strategies, with novel targets, can be discovered and deployed against a wide spectrum of human pathologies.

Address correspondence to: Rakesh K. Jain, Edwin L. Steele Laboratory, Department of Radiation Oncology, 100 Blossom Street,
Boston, Massachusetts 02114, USA. Phone: (617) 726-4083; Fax: (617) 724-1819; E-mail: jain@steele.mgh.harvard.edu.

1. Lammert, E., Cleaver, O., and Melton, D. 2001. Induction of pancreatic differentiation by signals from blood vessels. Science. 294:564-567.

2. LeCouter, J., et al. 2003. Angiogenesis-independent endothelial protection of liver: role of VEGFR-1. Science. 299:890-893.

3. Fukumura, D., et al. 2003. Paracrine regulation of angiogenesis and adipocyte differentiation during in vivo adipogenesis. Circ. Res. 93:88-97.

4. Hattori, K., et al. 2002. Placental growth factor reconstitutes hematopoiesis by recruiting VEGFR1(+) stem cells from bone-marrow microenvironment. Nat. Med. 8:841-849.

5. Shen, Q., et al. 2004. Endothelial cells stimulate self-renewal and expand neurogenesis of neural stem cells. Science. 304:1338-1340.

6. Carmeliet, P., and Jain, R.K. 2000. Angiogenesis in cancer and other diseases. Nature. 407:249-257.

7. Storkebaum, E., et al. 2004. Treatment of motoneuron degeneration by intracerebroventricular delivery of VEGF in a rat model of ALS. Nat. Neurosci. 8:85-92.

8. Donovan, M.J., et al. 2000. Brain derived neurotrophic factor is an endothelial cell survival factor required for intramyocardial vessel stabilization. Development. 127:4531-4540.

9. Lu, X., et al. 2004. The netrin receptor UNC5B mediates guidance events controlling morphogenesis of the vascular system. Nature. 432:179-186.

10. Kermani, P., et al. 2005. Neurotrophins promote revascularization by local recruitment of $\mathrm{TrkB}^{+}$endothelial cells and systemic mobilization of hematopoietic progenitors. J. Clin. Invest. 115:653-663. doi:10.1172/JCI200522655.

11. Hattori, K., et al. 2001. Vascular endothelial growth factor and angiopoietin-1 stimulate postnatal hematopoiesis by recruitment of vasculogenic and hematopoietic stem cells. J. Exp. Med. 193:1005-1014.

12. Hanahan, D., Lanzavecchia, A., and Mihich, E. 2003. Fourteenth Annual Pezcoller Symposium: the novel dichotomy of immune interactions with tumors. Cancer Res. 63:3005-3008.

13. de Bont, E.S., et al. 2001. Mobilized human
CD34+ hematopoietic stem cells enhance tumor growth in a nonobese diabetic/severe combined immunodeficient mouse model of human nonHodgkin's lymphoma. Cancer Res. 61:7654-7659.

14. Janowska-Wieczorek, A., et al. 1999. Growth factors and cytokines upregulate gelatinase expression in bone marrow CD34(+) cells and their transmigration through reconstituted basement membrane. Blood. 93:3379-3390.

15. Conejo-Garcia, J.R., et al. 2004. Tumor-infiltrating dendritic cell precursors recruited by a betadefensin contribute to vasculogenesis under the influence of Vegf-A. Nat. Med. 10:950-958.

16. Yang, L., et al. 2004. Expansion of myeloid immune suppressor $\mathrm{Gr}+\mathrm{CD} 11 \mathrm{~b}+$ cells in tumor-bearing host directly promotes tumor angiogenesis. Cancer Cell. 6:409-421.

17. Rajantie, I., et al. 2004. Adult bone marrow-derived cells recruited during angiogenesis comprise precursors for periendothelial vascular mural cells. Blood. 104:2084-2086.

18. Tateishi-Yuyama, E., et al. 2002. Therapeutic angiogenesis for patients with limb ischaemia by autologous transplantation of bone-marrow cells: a pilot study and a randomised controlled trial. Lancet. 360:427-435.

19. De Palma, M., Venneri, M.A., Roca, C., and Naldini, L. 2003. Targeting exogenous genes to tumor angiogenesis by transplantation of genetically modified hematopoietic stem cells. Nat. Med. 9:789-795.

20. Jiang, Y., et al. 2002. Pluripotency of mesenchymal stem cells derived from adult marrow. Nature. 418:41-49.

21. Kubo, H., and Alitalo, K. 2003. The bloody fate of endothelial stem cells. Genes Dev. 17:322-329.

22. Houghton, J., et al. 2004. Gastric cancer originating from bone marrow-derived cells. Science. 306:1568-1571.

23. Direkze, N.C., et al. 2004. Bone marrow contribution to tumor-associated myofibroblasts and fibroblasts. Cancer Res. 64:8492-8495.

24. Garcia-Barros, M., et al. 2003. Tumor response to radiotherapy regulated by endothelial cell apoptosis. Science. 300:1155-1159.

25. Hurwitz, H., et al. 2004. Bevacizumab plus irinotecan, fluorouracil, and leucovorin for metastatic colorectal cancer. N. Engl. J. Med. 350:2335-2342. 